\title{
Morphine reduced perceived anger from neutral and implicit emotional expressions
}

*Guro E. Løseth ${ }^{1}$, Marie Eikemo ${ }^{2,3}$, Peder Isager ${ }^{1}$, Jostein Holmgren ${ }^{1}$, Bruno Laeng $^{1}$, Vigdis Vindenes ${ }^{4}$, Trine Hjørnevik ${ }^{2}$, Siri Leknes ${ }^{1}$

${ }^{1}$ Department of Psychology, University of Oslo, Norway

${ }^{2}$ Department of Diagnostic Physics, Division of Radiology and Nuclear Medicine, Oslo University Hospital, Norway

${ }^{3}$ Division of Mental Health and Addiction, Oslo University Hospital, Norway

${ }^{4}$ Department of Forensic Medicine, Oslo University Hospital, Norway

* Corresponding Author Information

Guro Engvig Løseth

Department of Psychology

University of Oslo

Forskningsveien $3 \mathrm{~A}$

0373 Oslo

Postal address: Postbox 1094 Blindern, 0317 Oslo, Norway

Email: g.e.loseth@psykologi.uio.no 


\begin{abstract}
The $\mu$-opioid system modulates responses to pain and psychosocial stress and mediates non-social and social reward. In humans, the $\mu$-opioid agonist morphine can increase overt attention to the eyeregion and visual exploration of faces with neutral expressions. However, little is known about how the human $\mu$-opioid system influences sensitivity to and appraisal of subtle and explicit cues of social threats and reward. Here, we examined the effects of selective $\mu$-opioid stimulation on perception of anger and happiness in faces with explicit, neutral or implicit emotion expressions. Sixty-three healthy adults ( 32 females) attended two sessions where they received either placebo or $10 \mathrm{mg}$ per oral morphine in randomized order under double-blind conditions. Based on the known $\mu$-opioid reduction of pain and discomfort, as well as reports suggesting that the non-specific partial agonist buprenorphine or the non-specific antagonist naltrexone affect appraisal of social emotional stimuli, we hypothesised that morphine would reduce threat sensitivity and enhance perception of happy facial expressions. While overall perception of others' happiness was unaffected by morphine treatment, morphine reduced perception of anger in stimuli with neutral and implicit expressions without affecting perception of explicit anger. This effect was statistically unrelated to gender, subjective drug effects, mood and autism trait measures. The finding that a low dose of $\mu$-agonist reduced the propensity to perceive anger in photos with subtle facial expressions is consistent with the notion that $\mu$-opioids mediate social confidence and reduce sensitivity to threat cues.
\end{abstract}




\section{Introduction}

All social animals need to navigate an environment rich in social cues. Recognizing others' emotional expressions and successfully interpreting their intentions is necessary for adaptive social interactions. Accordingly, the brain devotes a large proportion of neurons to the processing of important social stimuli, such as the face and eyes of others (Itier and Batty, 2009). Inattention to social cues, as in autism spectrum disorder, is associated with poor social functioning (Chevallier et al., 2012). However, heightened vigilance to social signals could also be maladaptive. For instance, people with social anxiety display heightened sensitivity to subtle threat cues such as masked facial expressions of anger (Mogg and Bradley, 2002).

The emotional and motivational state of the individual affects which cues take priority and receive the most attention. The endogenous opioid system modulates both emotional-motivational state and responses to appetitive and aversive stimuli across species. Activation of the $\mu$-opioid receptors (MOR) is known to reduce pain and distress (Hsu et al., 2013; Leknes and Tracey, 2008; Lutz and Kieffer, 2013; Løseth et al., 2014). In the absence of aversive cues, MOR activation increases motivation and positive affective responses to rewards (Berridge and Kringelbach, 2013; Løseth et al., 2014). Rodent research has shown that the MOR system is also central for resolving conflicts between rewards and threats, e.g. dampening pain to enable approach of a valuable reward (Dum and Herz, 1984; Fields, 2006). In the social domain, MOR drugs enhance rodents' reward from social interaction (Trezza et al., 2011; Vanderschuren et al., 1996) and facilitate social encounters by dampening the normally occurring motivation to first explore new surroundings (Vanderschuren et al., 1995a, b), which could be linked to dampened anxiety or sensitivity to environmental threat cues (Panksepp et al., 1985)

Less is known about the $\mu$-opioid system's role in the modulation of reactions to social cues in humans. The limited available evidence from human research points to similar MOR modulation of social interest and vulnerability to threat. For instance, a previous report from our laboratory 
(Chelnokova et al., 2014) showed that motivation to view pictures of attractive faces with neutral expressions increased after treatment with the MOR agonist morphine compared to the opioid antagonist naltrexone. The two drugs also oppositely modulated time spent looking at the eye region as well as visual exploration of these faces as a whole, consistent with $\mu$-opioid enhancement of social interest (Chelnokova et al., 2016). Since morphine shows high affinity only to MORs, we interpret these bidirectional drug effects as primarily MOR-mediated. Naltrexone is an opioid antagonist, primarily at the $\mu$ - and $\mathrm{k}$-opioid receptors (KORs). Another commonly used drug in the human literature, buprenorphine, is a partial MOR agonist and KOR antagonist; accordingly, several related findings in the human literature may result from a combination of effects on MOR and KOR. Interestingly, animal studies suggest that KOR activation typically inhibits reward and promotes aversive states, whereas blocking KOR can dampen anxiety (Lutz and Kieffer, 2013).

A series of psychopharmacological studies using buprenorphine have yielded results consistent with a) increased sensitivity to positive social cues and $b$ ) reduced perception of threatening cues. For instance, compared to placebo buprenorphine improved short-term spatial memory for happy faces (Syal et al., 2015), increased ratings of positivity in response to pictures with social content and decreased perception of social rejection during a simulated social rejection task (Bershad et al., 2016). In addition, buprenorphine attenuated physiological stress responses to a psychosocial stress task (Bershad et al., 2015), selectively impaired recognition of fearful facial expressions in one study (Ipser et al., 2013), and reduced initial visual attention to fearful facial expressions in another (Bershad et al., 2016). The observed reduction in sensitivity and attention towards fear cues aligns well with evidence from other human studies using the MOR and KOR antagonists naloxone and naltrexone (but see Wardle et al., 2016). Indeed, naloxone enhanced acquisition of conditioned fear in humans (Eippert et al., 2008), a finding recently replicated and extended to fear conditioning induced solely through observation (social threat learning; Haaker et al., 2017). Another recent study measured implicit facial mimicry to happy faces and interpreted results as an indication of lowered interest in positive social interaction (Meier et al., 2016). 
While fearful facial expressions can communicate impending threats from the environment, angry facial expressions can act as a specific threat cue, i.e. indicating that an individual might become dangerous or violent. Happy facial expressions on the other hand signal potential for rewarding interaction. In fact, just looking at pictures of happy faces can be rewarding in itself (Spreckelmeyer et al., 2009). In the present study, we examine the effects of the preferential MOR agonist morphine on perception of explicit and implicit expressions of happiness and anger. A low dose of morphine (10 mg per oral) was chosen to mimic endogenous MOR activation with minimal subjective side effects (as in Chelnokova et al., 2014; and Eikemo et al., 2016). We hypothesised that morphine would dampen perception of anger and increase perception of happiness in the faces of others. We also tested the competing hypothesis that morphine would improve detection of implicit facial expressions regardless of valence. This was based on our previous finding that morphine increased visual exploration of faces and time spent looking at the eye-region (Chelnokova et al., 2016). The peptide oxytocin, which also increases gaze to the eyes, is found to consistently improve emotion recognition (Leppanen et al., 2017). Indeed, we have previously found (Leknes et al., 2013) that intranasal oxytocin improved sensitivity to implicit expressions of anger and happiness in the same set of face stimuli used in the present study. Consistent with the finding that oxytocin improved emotion recognition the most in people with autistic traits (Bartz et al., 2010), Leknes et al. (2013) also reported that the greatest oxytocin-related improvement in emotion perception occurred in participants with a lower ability to perceive subtle emotional cues in the placebo condition. Based on this, as well as mounting evidence indicating that opioid dysregulation underpins the blunted social reward associated with autism (Pellissier et al., 2017), we explored whether morphine would enhance emotion perception more in participants with higher autistic traits.

\section{Methods and materials}

\subsection{Study design}


The within-subject, double-blind design consisted of two sessions where participants received $10 \mathrm{mg}$ per oral morphine or placebo in counterbalanced order before completing tasks assessing reward responses and emotion perception. The 'emotion perception' task was part of a larger study investigating effects of enhanced $\mu$-opioid system activation on various behavioural reward measures and BOLD signal activity measured with fMRI (data to be reported elsewhere). The emotion perception task was completed outside of the MR-scanner at 135 minutes after ingestion of drug/placebo.

\subsection{Participants}

Sixty-three healthy adult participants (32 females, 31 males) aged 19-45 years (mean age $27, \mathrm{SD}=5$ ) completed testing with morphine and placebo in separate sessions. Sixty-eight participants were recruited through flyers and online advertisement and underwent a phone-assisted screening interview prior to inclusion. Exclusion criteria were ongoing or self-reported history of major psychiatric illness including depression and drug- or alcohol use disorder; no history of or ongoing prolonged pain condition; no current use of medication (antihistamines and contraceptives exempt), and no multiple complex allergies. Other exclusion criteria were pregnancy, a history of prolonged opioid medication, single- or repeated use of any strong opioids the last two years, and use of drugs containing codeine or other mild opioids during the last four months. All participants had normal or corrected-to-normal vision. Data from five participants was excluded: One participant received placebo both sessions; four were not able to return for their second session. See Table 1 for characteristics of the final sample of 63 participants.

Table 1. Participant characteristics*

\begin{tabular}{llllllll}
\hline & total & \multicolumn{2}{l}{ female } & male \\
& $n=63$ & \multicolumn{3}{c}{$n=32$} & & $n=31$ & \\
\hline Age & 27 & \pm 5.0 & 27 & \pm 3.7 & 27 & \pm 6.2 \\
Weight $(\mathrm{kg})$ & 74 & \pm 11.7 & 66 & \pm 7.3 & 81 & \pm 10.5 \\
Height $(\mathrm{cm})$ & 176 & \pm 9.1 & 170 & \pm 6.8 & 182 & \pm 6.4 \\
BMI & 24 & \pm 2.8 & 23 & \pm 2.5 & 24 & \pm 2.9 \\
\hline
\end{tabular}


*all values are means \pm standard deviation

Self-report data revealed that 23 of the 32 female participants used hormonal contraceptives. Of the remainder of the female sample, six confirmed that both test sessions fell within the same phase of the hormonal cycle; three were unable to estimate number of days since their last ovulation. Self-reported recreational drug and alcohol was recorded as part of the pre-test screening using selected items from AUDIT and DUDIT (Alcohol/Drug Use Disorders Identification Test) (Berman et al., 2005; Saunders et al., 1993). See Table 2 for overview. Participants were instructed not to consume alcohol for 24 hours before testing, to abstain from caffeine and tobacco for a minimum of one hour before the test session commenced, and advised not to drive or operate heavy machinery for six hours after drug administration. All participants were asked to eat a few hours or less before testing, and were offered a light meal or snack if they reported being hungry upon arrival.

\begin{tabular}{|c|c|c|c|c|}
\hline Alcohol use & \multicolumn{2}{|c|}{ Range } & \multicolumn{2}{|c|}{ Average $(S D)$} \\
\hline Units per week* & \multicolumn{2}{|l|}{$0-40$} & \multicolumn{2}{|c|}{$8(7)$} \\
\hline \multirow[t]{2}{*}{ Tobacco use } & \multicolumn{2}{|l|}{ Daily } & \multicolumn{2}{|c|}{ Occasionally } \\
\hline & $\%$ & $n$ & $\%$ & $n$ \\
\hline Cigarettes & $8 \%$ & 5 & $6 \%$ & 4 \\
\hline Snuff tobacco 'snus' & $16 \%$ & 10 & $11 \%$ & 7 \\
\hline \multirow[t]{2}{*}{ Drug use } & \multicolumn{2}{|c|}{ Life time } & \multicolumn{2}{|c|}{ Last year } \\
\hline & $\%$ & $n$ & $\%$ & $n$ \\
\hline Cannabis & $78 \%$ & 49 & $40 \%$ & 25 \\
\hline Amphetamines ${ }^{1}$ & $16 \%$ & 10 & $5 \%$ & 3 \\
\hline Cocaine $^{2}$ & $26 \%$ & 17 & $16 \%$ & 10 \\
\hline Opiates $^{3}$ & $2 \%$ & 1 & $0 \%$ & 0 \\
\hline Hallucinogens ${ }^{4}$ & $9 \%$ & 14 & $10 \%$ & 6 \\
\hline Solvents ${ }^{5}$ & $2 \%$ & 1 & $0 \%$ & 0 \\
\hline GHB and other drugs ${ }^{\ominus}$ & $17 \%$ & 11 & $3 \%$ & 2 \\
\hline
\end{tabular}

Table 1. Information about alcohol, tobacco and recreational drug use. Percentage (\%) and number of participants ( $n$ ). Categories also include: ${ }^{1}$ Methamphetamine, phenmetrazine, khat, betel nut; ${ }^{2}$ crack, freebase, coca leaf; ${ }^{3}$ heroin and opium; ${ }^{4} \mathrm{MDMA}, \mathrm{LSD}$, mescaline, peyote, $\mathrm{PCP}$, psilocybin; ${ }^{5}$ Thinner, trichloroethylene, gas, glue; ${ }^{6} \mathrm{GHB}$, anabolic steroids, nitrous oxide, poppers (amyl nitrate) and anticholinergic drugs.

\footnotetext{
* one Norwegian unit equals 15 grams of alcohol
} 


\subsection{Procedures}

Experimental procedures were approved by the Regional Ethics Committee (2011/1337/REK sør- $\varnothing$ st D). Participants were tested on two different days with a minimum inter-session interval of 48 hours. Each session lasted approximately 3 hours. After giving informed and written consent, participants completed a questionnaire measuring subjective state, as well as an MRI safety questionnaire. Participants then received tablets containing either $10 \mathrm{mg}$ morphine or placebo. During the $60-$ minute interval between drug administration and test onset, participants watched a nature program of choice for 30 minutes, before completing a trial version of the fMRI tasks. Participants were then installed in the MR-scanner and answered the second subjective state questionnaire, before completing a battery of tasks measuring monetary incentive delay and regulation of craving in response to food images. After exiting the scanner, participants were offered a short break and a blood sample was collected (data to be reported elsewhere). At approximately $135 \mathrm{~min}$ after drug ingestion, participants moved to a separate room where they completed the emotion perception task. At 155 min post drug administration, participants completed subjective measures for the third and last time and performed a motor coordination task. Towards the end of the second session, participants completed a set of questionnaires and were asked to guess the identity of the drug given each session, before debriefing. Participants were reimbursed on average 340 NOK (about 40 USD) for their participation, +/- 20 NOK based on their performance on the monetary incentive delay task.

\subsection{Drug administration}

Morphine is an opioid agonist with high affinity to MOR, and the most widely chosen analgesic (Vindenes et al., 2006). To enhance MOR activation with minimal subjective effects, we administered 10 mg per oral morphine tablets (Morfin ${ }^{\circledR}$, Nycomed Pharma, Asker, Norway). Maximal effect of oral morphine is reached 1-2 hours after administration $\left(T_{\max }\right)$ at peak concentration in blood, half-life is 2-4 hours and the average bioavailability is $30-40 \%$ (Lugo and Kern, 2002). A test interval of 60-180 min after drug administration was chosen to ensure relatively high and stable levels of morphine 
throughout the session. Placebo tablets were sugar-free cherry-flavoured breath mints matched to the morphine tablets in both size and shape. Tablets were administered by handing the participant a pre-prepared opaque lidded beaker, preventing both the participant and the experimenter from visually inspecting the content. The beaker always contained two tablets, since one breath mint was always added to the morphine dose to avoid taste recognition as in our previous reports (Chelnokova et al., 2016; Eikemo et al., 2017). Participants were instructed to open the beaker without looking and swallow the tablets immediately with some water. To further reduce expectancy, participants were told they might receive an opioid agonist (morphine), antagonist (naltrexone) or placebo at any session.

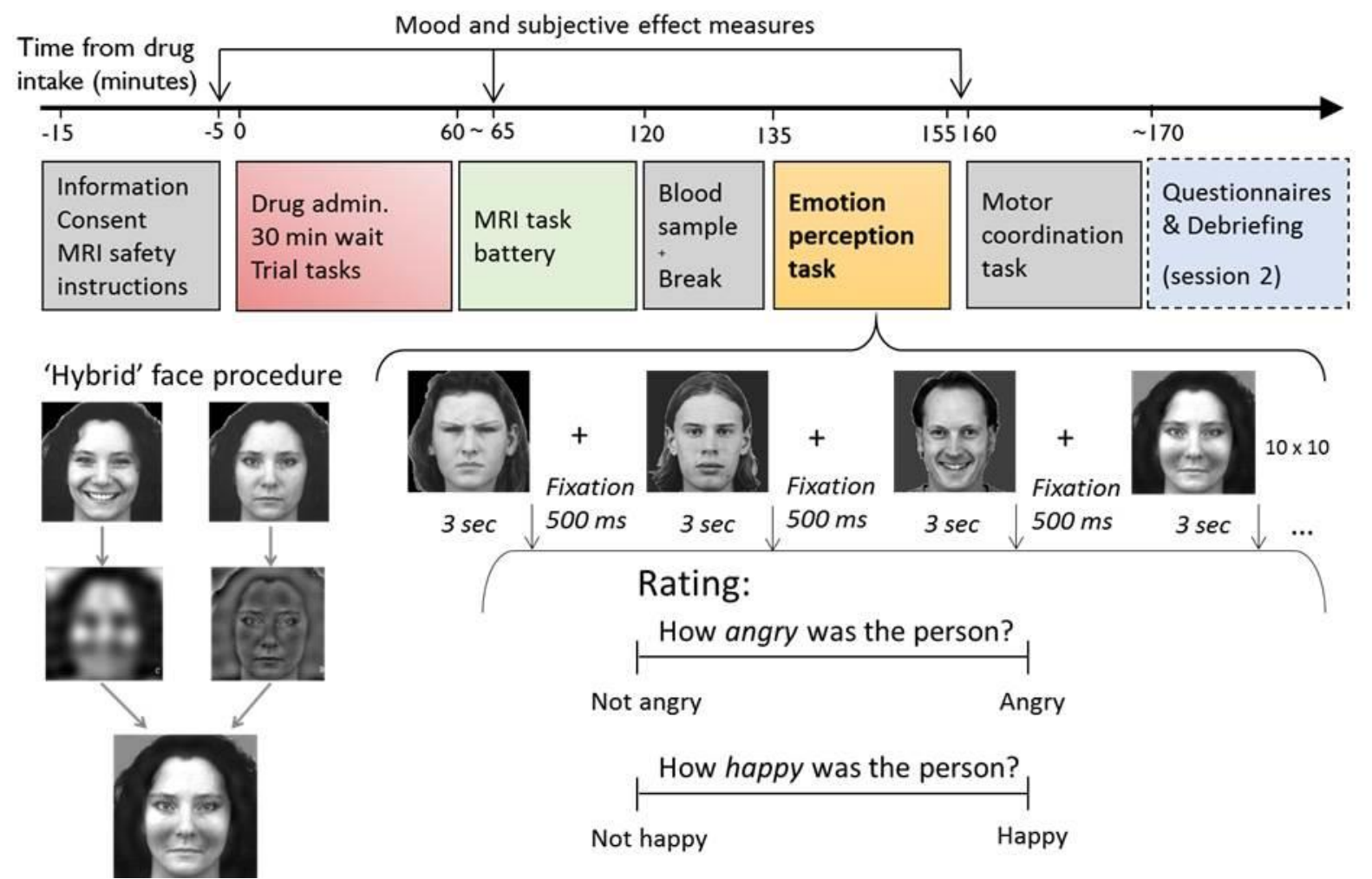


Figure 1: Overview of study design. Participants received $10 \mathrm{mg}$ morphine or placebo and then underwent a task protocol consisting of tasks administered in an MR-scanner, and the emotion perception task reported here. The emotion perception task consisted of images depicting faces with five expression types: explicit happiness; implicit (hybrid) happiness; neutral; implicit (hybrid) anger; explicit anger. Implicit expressions contained high-frequency visual information from a neutral expression and low-frequency visual information from the same person expressing either anger or happiness. These stimuli are perceived as neutral, but have previously been shown to evoke a core impression such that faces containing implicit happiness were perceived as more friendly than faces containing implicit anger, fear or sadness (Laeng et al., 2010). Participants rated perceived anger and perceived happiness for each image. Images were adapted from the Karolinska Directed Emotional Faces KDEF (CD-ROM), by D. Lundqvist, A. Flykt, \& A. Ohman, 1998, Stockholm, Sweden: Department of Clinical Neuroscience, Psychology section, Karolinska Institutet. Reprinted with permission. Time since drug administration is displayed in minutes at the beginning of each block. The task order was identical for all subjects in both sessions.

\subsection{Control measures}

\subsubsection{Subjective measures}

Mood and subjective drug effects were assessed at pre-drug baseline and at 60 and 155 minutes post-drug, using a 21-item checklist developed locally as described in previous publications (Chelnokova et al., 2014; Eikemo et al., 2016). Participants indicated their responses on 11-point visual analogue scales (VAS), with the anchors 'Not at all' and 'Very much'. For the pre-drug baseline and the post-scanning measures, the questionnaire was presented on a computer screen using MatLab software (version 7.10.0. Natick, Massachusetts: The MathWorks Inc., 2010). The subjective measure questionnaire administered in the MR scanner was presented with a similar visual design using E-prime 2.0 (Psychology Software Tools, Pittsburgh, PA).

\subsubsection{Motor coordination task}

Potential drug effects on motor function and alertness were assessed each session using an eye-hand coordination test administered after the emotion perception task, at approximately 160 min post drug (Bradykinesia Akinesia Incoordination task, Giovannoni et al., 1999). Using their dominant index finger, participants were instructed to alternate as quickly and accurately as possible between pressing two keys placed $15 \mathrm{~cm}$ apart on a standard keyboard for 60 seconds. As in our previous 
reports (Chelnokova et al., 2014; Eikemo et al., 2016), we used the Dysmetria score (DS), a weighted index of speed and accuracy reflecting overall task performance (Giovannoni et al., 1999), to compare motor function across drug conditions. Data from twelve participants (six female, six male) were incomplete due to a computer error, leaving a final $N$ of 51 for this task.

\subsection{Emotion perception task}

Perception of anger and happiness in response to pictures of faces was measured using a task adapted from Leknes and colleagues, previously showed to be sensitive to effects of intranasal oxytocin (Leknes et al., 2013). In each task, participants viewed black-and-white images of actors (20 males, 20 females) from the Karolinska Directed Emotional Faces database (Calvo and Lundqvist, 2008; Lundqvist et al., 1998), displaying explicitly angry, explicitly happy and neutral expressions. In addition, two implicitly emotional images (happy hybrid and angry hybrid) were created by merging low-passed spatial frequency images $\left(\mathrm{SF} \leq 6^{\circ}\right)$ of angry or happy images with the middle and high spatial frequencies $\left(\mathrm{SF} \geq 7^{\circ}\right.$ ) of a neutral image of the same face, as described by Laeng and colleagues (Laeng et al., 2010) and illustrated in Figure 1. These implicitly emotional images have been shown to be categorised as neutral despite containing a specific emotional expression within the low spatial frequencies of the photo (Laeng et al., 2010). Furthermore, participants were unable to distinguish happy hybrid, angry hybrid and neutral images in a forced-choice detection task, confirming that participants were indeed unaware of the presence of a specific emotion. Nevertheless, the emotional content in the hybrid images affected the core impression of the depicted subject at a subliminal level, with participants rating happy hybrid faces as friendlier and angry hybrid faces as more unfriendly than neutral faces (Laeng et al., 2010).

As in Leknes et al (2013), a total of 200 images from the original study by Laeng et al. (2010) were used. The same 40 models were shown with five facial expressions: explicitly happy, implicitly happy (happy hybrid), neutral, implicitly angry (angry hybrid) and explicitly angry. Each image was presented for 3 seconds, preceded by a fixation cross displayed for $500 \mathrm{~ms}$. One stimulus set of 100 
unique images of all 40 individuals was presented each session in a pseudo-randomised order according to the following rules: never more than two consecutive images of the same expression or person; all types of expressions presented within each 10-stimulus block; at least two images of each gender within each block. Images $(8 \times 8 \mathrm{~cm})$ were presented on a computer monitor situated $57 \mathrm{~cm}$ in front of the participant in a windowless room with constant artificial lighting. Stimuli were smaller in size and presented on a smaller screen than in Laeng et al. (2010) and Leknes et al. (2013), and distance between participants' eyes and computer monitor was adjusted accordingly to ensure that the pictures covered the same area of visual field. Visual stimuli and rating scales were presented using E-prime 2.0 (Psychology Software Tools, Inc). No images were repeated across sessions. Ratings of perceived happiness and anger of the face stimuli were measured using two separate 11-point visual-analogue scales (VAS) ranged from 0-10, one for each mood, both displayed after each image in a pseudo-randomised order: A) How happy was the person? (anchors: Not happy-happy); B) How angry was the person? (anchors: Not angry - angry).

Five participants were by mistake presented the same stimulus set at both sessions. All statistical analyses were conducted both with and without data from these participants, to ensure that models were not biased by any recognition effects affecting these participants' responses.

\subsection{Autism trait measure}

We used the Autism Spectrum Quotient (AQ; Baron-Cohen et al., 2001b) to measure variance in baseline social competencies in our participant sample. The 50-items questionnaire was administered as an online survey, with individualized links sent to participants after they had completed both experiment sessions. AQ (potential score range $0-50$ ) was calculated according to standard procedures. Participants were instructed to complete the questionnaire within one week of participation. However, five participants (four female, one male) did not complete the questionnaire and data from these were therefore excluded from statistical analyses where $A Q$ was included.

\section{Statistical analyses}




\subsection{Control measures}

\subsubsection{Subjective measures}

Statistical analysis was run using SPSS version 24 (IBM). Subjective state ratings from the two postdrug measurement points were baseline-corrected and aggregated before drug effects on each measure were assessed with repeated-measures analyses of variance (rmANOVA). In cases where sphericity assumptions were violated, the Greenhouse-Geisser correction was used. Any subjective measure upon which drug treatment had a significant effect were added as control covariates in analyses of the main outcome variables.

\subsubsection{Motor coordination}

Potential drug effect on motor coordination was assessed with a paired sample t-test comparing dysmetria scores from the two drug conditions.

\subsection{Emotion perception ratings}

Ratings of perceived happiness and perceived anger were analysed separately with linear mixed models (LMMs) using the MIXED command in SPSS (version 24, IBM). The model selection included combinations of the following fixed effects: stimulus type, drug, stimulus type*drug interaction, session number, drug*session interaction, participant gender, age, weight, and stimulus gender. To adjust for dependencies in the data related to individual characteristics of each single item (picture) or participant, all LMMs had a crossed random effect structure which included by-item and bysubject random intercepts, as well as a by-subject random slope for stimulus type. The random effects variance-covariance matrix had a variance-components structure. Interaction effects between stimulus type and drug were explored with planned pairwise post-hoc comparisons contrasting ratings between drug conditions for each stimulus type.

Model selection and evaluation. Fixed factors relating to the experimental design, recruitment and counterbalancing (such as stimulus type, drug, stimulus type*drug interaction and session number), 
were always kept in the main LMMs. Aside from these variables we aimed for parsimonious models. The remaining covariates and interactions between fixed factors not relating to our hypotheses were removed when they did not significantly affect the outcome and/or did not improve model fit as indexed by a Bayesian Information Criteria (BIC) reduction of 2 or more. Adding the random terms for by-subject intercept and slopes improved all LMMs as indicated by lower BIC and significant Wald $Z$ statistics. The final models selected for each analysis are described in the result section.

\section{Exploratory analyses of gender effects and interactions. Gender effects (participant} gender and interactions with drug and stimulus type) were explored in separate LMMs. To control for weight differences between gender groups, participant weight was included as a fixed covariate. Otherwise, the LMMs were built identically to the final main models.

Estimated Means and Standard Errors. All means and standard errors reported for ratings of anger and happiness are estimated means and SEMs from the final models. In graphs where groups are compared (i.e. gender) between-subject standard error of the mean (SEMs) from the models are provided. For analyses where within-subject contrasts are of primary interest (i.e. drug or stimulus type), within-subject SEMs were calculated from raw scores.

\subsubsection{Individual differences in sensitivity to implicit emotional expressions}

\section{Assessing emotion sensitivity}

To investigate whether the effects of morphine would vary as a function of individual sensitivity to the implicit emotional expressions conveyed by the hybrid images, an emotion sensitivity score (ES) was calculated for each participant per session (as in Leknes et al. 2013). In short, a participant's ES was the average difference in (i) perceived happiness and (ii) perceived anger between hybrid angry images and hybrid happy images. Participants who on average rated hybrid angry images as angrier and less happy than hybrid happy images, would receive a high ES. A low ES reflected either no reliable difference in ratings between the two hybrid image types, or a tendency to perceive more of the emotion incongruent with the implicit emotional expression. The ES from the placebo session 
served as a baseline trait measure. Effects of morphine on emotion sensitivity were assessed in a LMM, with drug and session as fixed factors, and by-subject intercept inluded as a random factor.

\section{Assessing relationship between emotion sensitivity and autistic traits}

Healthy individuals with normal intelligence that are higher in autistic traits have been reported to show impaired identification of emotional facial expressions (Poljac et al., 2013) and reduced social sensitivity (Baron-Cohen et al., 2001a). An inverse correlation between the ES measure and AQ (autistic traits) was hypothesized by Leknes et al (2013), such that individuals with high ES would tend to have lower $\mathrm{AQ}$ - i.e. fewer traits associated with autism. Here, we tested this hypothesis using a paired bivariate correlation analysis (two-tailed) on baseline (placebo) ES and AQ ( $n=58$ ).

Finally, to explore whether $A Q$ moderated effects of morphine on emotion sensitivity, AQ was added as a fixed covariate in an LMM, with drug and session as fixed factors and by-subject intercept included as random factor $(n=58)$.

\section{Results}

\subsection{Control measures}

\subsubsection{Drug blinding}

In a debriefing interview conducted upon completion of the last session, $40 \%$ guessed correctly which session they had received an active drug and $55 \%$ of participants correctly guessed which session they had received placebo, indicating successful blinding. Only $20 \%$ of participants guessed that the active drug they had received was morphine, the remainder believed they had received naltrexone.

\subsubsection{Subjective measures}

As in our previous studies (Chelnokova et al., 2014; Eikemo et al., 2016), 10 mg morphine (M) had minimal effect on subjective measures compared to placebo (P). Repeated measures ANOVAs 
revealed a significant effect of drug only on ratings of 'Dry Mouth' (Mean (SEM): $M=1.049$ (.311), $\mathrm{P}=$ $-.461(.318), F_{1,114.005}=12.580, p=.001$, partial $\left.\eta 2=.204\right)$. No other individual subjective measures were significantly affected by drug condition, even without controlling for multiple comparisons. Notably, drug condition did not significantly affect mood, feelings of being high or experience of drug effect. The largest mean effect of morphine was 1.2 on the 11-point VAS scale for any one item, and the average difference between drug conditions on changes in subjective state from baseline was

0.31. See Table 3 for a summary of the results.

Table 3 Baseline-corrected mean (SEM) ratings of subjective measures

\begin{tabular}{lccccc}
\hline Ratings of feeling ... & \multicolumn{2}{l}{ Placebo } & \multicolumn{2}{l}{ Morphine } & -value \\
\hline Good & -.502 & $(.176)$ & -.374 & $(.201)$ & .517 \\
Happy & -.306 & $(.156)$ & -.276 & $(.171)$ & .889 \\
Self-confident & -.201 & $(.176)$ & -.246 & $(.200)$ & .845 \\
Irritable & .428 & $(.202)$ & .266 & $(.186)$ & .519 \\
Anxious & .220 & $(.119)$ & -.053 & $(.240)$ & .289 \\
Discomfort muscles/joints & .505 & $(.237)$ & .488 & $(.210)$ & .953 \\
Nauseous & .383 & $(.158)$ & .219 & $(.166)$ & .479 \\
Dry mouth * & -.404 & $(.292)$ & .961 & $(.277)$ & $.000 *$ \\
Hungry & 2.313 & $(.277)$ & 2.591 & $(.262)$ & .337 \\
Red/warm face & .358 & $(.193)$ & .521 & $(.277)$ & .610 \\
Dizzy & .454 & $(.225)$ & 1.032 & $(.275)$ & .092 \\
Blunted & .293 & $(.260)$ & .907 & $(.303)$ & .088 \\
Numb & .893 & $(.207)$ & 1.036 & $(.225)$ & .626 \\
Not myself & .842 & $(.256)$ & .500 & $(.263)$ & .392 \\
High & 1.089 & $(.217)$ & 1.291 & $(.249)$ & .512 \\
Tired & .991 & .240 & 1.032 & .273 & .910 \\
Spaced out & .767 & .210 & 1.268 & .294 & .182 \\
Drug effect & 1.742 & .253 & 2.221 & .293 & .183 \\
Liking drug effect & 3.937 & .224 & 3.779 & .254 & .568 \\
Disliking drug effect & 2.847 & .241 & 3.231 & .265 & .206 \\
Would take tablets again & 4.534 & .298 & 4.133 & .325 & .157 \\
\hline$*$ statisticaly significant & & & & &
\end{tabular}

* statistically significant effect of morphine treatment compared to placebo.

\section{coordination}


A two-tailed paired t-test of dysmetria scores $(N=51)$ indicated no significant difference in motor function between the morphine $($ mean $=1.161, S D=.28)$ and placebo $($ mean $=1.156, S D=.26)$ conditions; $t(50)=.202, p=.841$.

\subsubsection{Picture set}

Post-hoc t-tests confirmed that overall ratings of happiness and anger did not differ between the two unique picture sets to a statistically significant degree. For details, see Supplementary Materials.

\subsection{Emotion Perception}

Ratings of perceived anger and perceived happiness were analysed in separate LMMs. Ratings of 'Dry mouth' were added as a fixed control covariate during model selection, but did not contribute to model fit or change the overall pattern of results, and were thus not included in the final models. The final LMMs for both types of ratings included the following fixed effects: stimulus type, drug, stimulus type*drug, and session.

\subsubsection{Main effect of stimulus type on perception of happiness and anger}

The main effect of stimulus type was significant for both happiness $\left(F_{4,354.173}=383.248, p<.001\right)$ and anger ratings $\left(F_{4,389.842}=226.397, p<.001\right)$. As expected, ratings of perceived happiness were highest for the explicitly happy faces and lowest for the explicitly angry faces, while most anger was perceived in explicitly angry faces and least in explicitly happy faces. Pairwise comparisons between stimulus type categories suggest that the subtle emotional expressions in the hybrid pictures affected participants' perception of emotion. Overall, hybrid happy faces were rated as significantly happier than neutral faces (mean difference $=.507$, SEM $=.201, p=.012$ ) and angry hybrids (mean difference $=.710, \mathrm{SEM}=.210, p=.001)$. Hybrid angry faces were rated significantly angrier than the hybrid happy faces (mean difference $=.511$, SEM $=.238, p=.032$ ), but not significantly more angry than neutral faces (mean difference $=.168$, SEM $=.238, p=.479$ ).

\subsubsection{No significant effects of morphine on ratings of perceived happiness}


There was no significant main effect of drug condition on happiness ratings $\left(F_{1,12100.992}=3.187, p\right.$ $=.074)$, and no significant interaction between drug condition and stimulus type $\left(F_{4,12101.086}=1.630\right.$, $p=.164)$. Results are displayed in Figure $2 \mathrm{a}$. A significant effect of session emerged $\left(F_{1,12100.453}=\right.$ 39.572, $p=<.001$ ), with slightly higher overall ratings of perceived happiness reported in session one compared to session two (mean difference $=.158, \mathrm{SEM}=.025$ ). Also, there was a significant session*drug interaction $\left(F_{1,60.992}=5.908, p=.018\right)$. Morphine received at session one was associated with reduced perception of happiness compared to placebo (mean difference -.494, $p=.010$ ), while morphine received at session two was associated with increased perception of happiness compared to placebo (mean difference $.404, p=.034$ ). Removing the session*drug interaction from the models' fixed effect statement did not alter the pattern or statistical significance of the main effect of $d r u g$ or the drug*stimulus type interaction.

\subsubsection{Morphine significantly reduced ratings of perceived anger for neutral and hybrid} stimuli

There was a significant main effect of drug condition on perception of anger, with morphine reducing overall perception of anger compared to placebo (mean (SEM) M: 3.395 (.141); P: 3.522 (.141); $F_{1 \text {, }}$ $12105.014=17.538, p<.001)$. Pairwise comparisons revealed that the mean difference between morphine and placebo conditions was driven by morphine's effects on perception of the hybrid and neutral stimuli. Compared to placebo, morphine significantly reduced ratings of anger for happy hybrids $($ mean difference $=-.279$, SEM $=.068, p<.001$ ), neutral faces (mean difference $=-.154$, SEM $=.069, p=.025$ ) and angry hybrids (mean difference $=-.143$, SEM $=.068, p=.034$ ). Drug condition did not significantly affect anger ratings for explicitly angry or explicitly happy faces (mean differences $=$ explicitly angry faces: $\mathrm{P}<\mathrm{M}-.011, p=.876$; explicitly happy faces: $\mathrm{P}>\mathrm{M} .07, p=.295)$. Results are displayed in Figure $2 \mathrm{~b}$. A significant effect of session on anger ratings emerged $\left(F_{1,12104.303}\right.$ $=12.228, p=<.001$ ), with slightly higher overall ratings of perceived anger reported in session one compared to session two (mean difference $=.106, \mathrm{SEM}=.030$ ). The session*drug interaction was not 
significant for anger ratings $(F=2.784, p=.100)$, and the interaction term was thus not kept in the final model reported here.

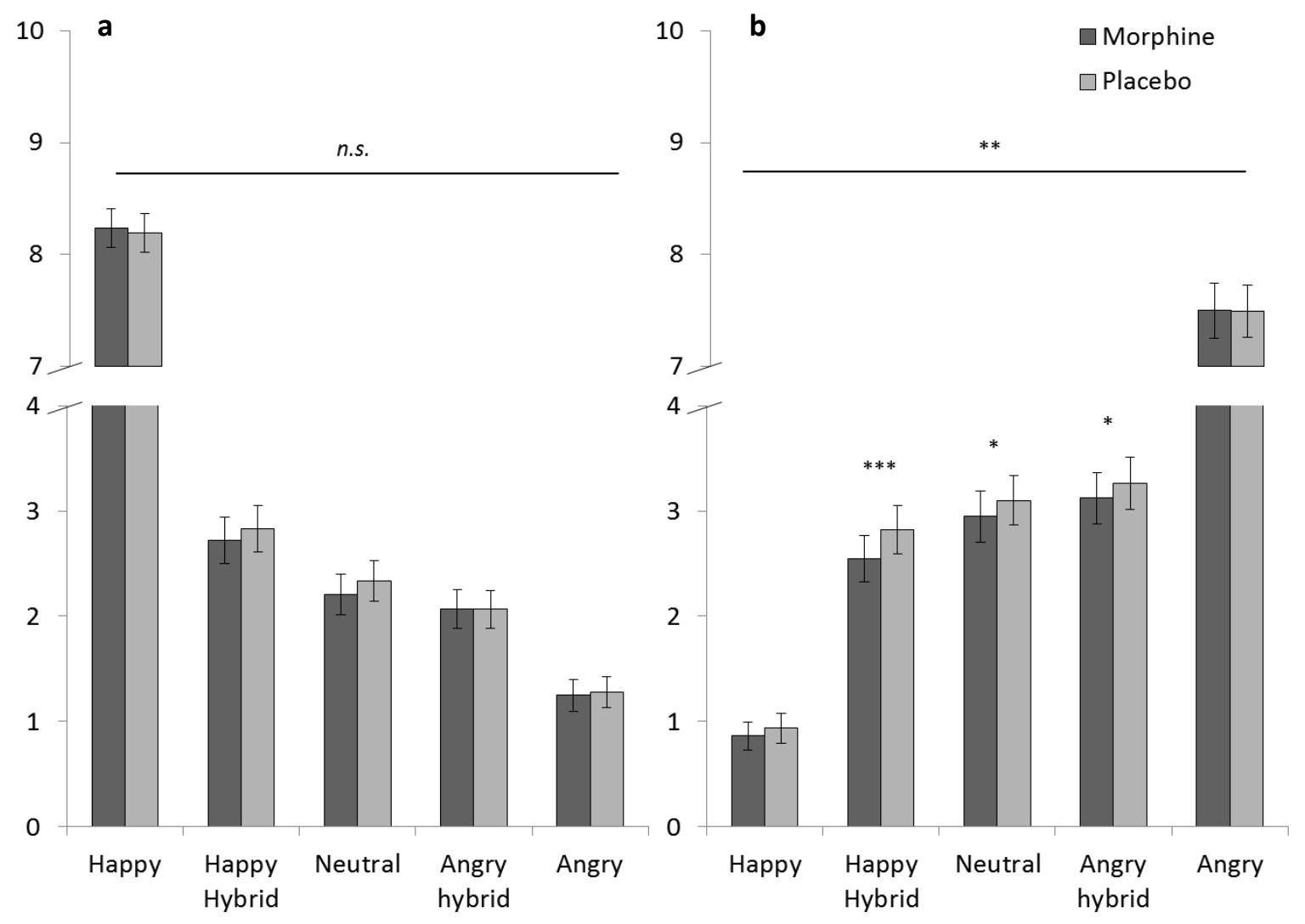

Figure 2: Effects of morphine on emotion perception. a) Ratings of perceived happiness were overall not significantly affected by morphine compared to placebo $(p=.074)$, and no significant interaction was found between drug category and stimulus type $(p=.164)$. b) Ratings of perceived anger were significantly lowered by morphine treatment compared to placebo $(p<.001)$. There was a significant interaction between drug category and stimulus type, with morphine inducing reductions in perceived anger for the hybrid happy $(p<.001)$, neutral $(p=.025)$ and hybrid angry $(p=.034)$ stimuli. Perception of anger in overtly angry or overtly happy stimuli was not significantly affected by drug condition.

\subsubsection{Gender effects}

Two LMMs exploring effects of gender on perception of happiness and anger respectively (participant gender, weight and interactions between gender and drug and stimulus type were added to each of the two LMMs used to assess drug effects on happiness and anger perception) revealed a significant main effect of participant gender on ratings of perceived happiness, reflecting a tendency for male participants to rate face stimuli as happier compared to female participants (male 
participants mean $=3.633, \mathrm{SEM}=.162 ;$ female participants mean $=3.013 ; \mathrm{SEM}=.159 ; F 1,59.994=$ $6.524, p=.013)$. There was no significant main effect of participant gender on anger ratings $\left(F_{1}, 60.003\right.$ $=.124, p=.726$ ). No significant interactions were found between participant gender and drug (anger ratings $F_{1}, 12100.506=.579, p=.447$; happiness ratings $F_{1}, 12096.328=.353, p=.553$ ) or participant gender and stimulus type (anger ratings $F_{4}, 243.868=1.310, p=.267$; happiness ratings $F_{4,} 244.240=$ $2.353, p=.055)$. The three-way interaction between participant gender, drug and stimulus type also failed to reach significance for both happiness and anger perception (largest $F=1.659$, smallest $p$ $=.157)$. The pattern of drug effects from the main analyses (displayed in Fig.2) was not changed in the LMMs where gender, weight and their interactions with drug were added to the models' fixed effects statement.

\subsubsection{Emotion sensitivity - not affected by morphine}

ES scores were calculated for all 63 participants and for each drug condition as described above, and analysed in a separate LMM with drug and session as fixed factors, and by-subject intercept inluded as a random factor. In the baseline (placebo) condition ES scores ranged between -.64 to 1.97, mean $=.58(\mathrm{SD}=.57)$, variance $=.323$. The LMM yielded no significant effect of $\operatorname{drug}(F 1,61=.056, p$ $=.814)$ or session $(F 1,61=.068, p=.795)$ on ES.

\subsubsection{Autistic traits - no correlation with emotion sensitivity found}

Fifty-eight of the participants (28 female, 30 male) completed the AQ questionnaire. AQ scores ranged between 4 and 32 on the 50 point scale, median $=14$, mean $=14.79$ (SD 5.67). Baseline (placebo) ES scores for this sample had a range of -.44 to 1.97 (mean $=.55$, SD .57). Contrary to our expectations, AQ did not significantly correlate with baseline ES (Pearson's $R=-.028, p=.835, n=58$ ).

\subsubsection{Autistic trait measures did not moderate effect of drug on emotion sensitivity}

The LMM exploring effects of $d r u g, A Q$ and $d r u g^{*} A Q$ interactions on emotion sensitivity scores (including drug and session as fixed factors, AQ score as a fixed covariate, and by-subject intercept as random factor) yielded no significant main effects or interactions (all $F^{\prime} s<0.7$, all $p^{\prime} s>$.8). 


\section{Discussion}

In this study, we investigated the effects of $\mu$-opioid stimulation with morphine on healthy humans' perception of anger and happiness in faces expressing emotions explicitly or implicitly. As hypothesised, we found that a non-sedative (10 mg per oral) dose of morphine decreased perception of anger compared to placebo. This effect was present for implicitly angry, neutral and implicitly happy facial expressions. Morphine did not significantly alter ratings of faces expressing explicit anger or happiness, however. Furthermore, we found no support for the hypothesis that morphine would enhance perception of others' happiness. These findings are broadly consistent with the known role of $\mu$-opioids in regulation of pain and negative affect (Levine et al., 1978). The lack of morphine-enhanced happiness perception however, differs from earlier reports that appraisal and motivation for positive stimuli are enhanced by MOR stimulation (Chelnokova et al., 2014; Syal et al., 2015).

The observed reduction in anger perception after $\mu$-opioid stimulation with morphine is in line with previous evidence of reduced sensitivity to social threat cues with the partial opioid agonist buprenorphine (Bershad et al., 2016; Ipser et al., 2013), and with studies showing enhanced fear learning after opioid blockade in humans (Eippert et al., 2008; Haaker et al., 2017). The reduced anger perception was not specific to stimuli containing cues of anger, and in fact, perception of emotion from explicitly angry faces was comparable across morphine and placebo conditions. Thus, morphine's effects on perception of a threatening social cue did not resemble a sort of 'social analgesia', in which perception of others' negative emotion is blunted across stimulus intensities. Instead, morphine reduced the proneness to perceive anger specifically for stimuli without explicit emotional expressions, i.e. expressions that left room for doubt. Ratings of these stimuli could therefore be more susceptible to subtle differences in core impressions \{laeng,2010\} due to morphine treatment than the faces showing explicit anger or happiness expressions. 
We found no evidence supporting the hypothesis that morphine would enhance perception of happiness. Considering the sample size of 63 participants and the within-subject design, together with a non-significant trend in the opposite direction, we tentatively reject our hypothesis regarding perception of others' positive emotion. Nevertheless, other evidence has indicated increased social reward responses with MOR agonism in humans and rodents (Chelnokova et al., 2014; Syal et al., 2015; Trezza et al., 2011; Vanderschuren et al., 1996; Wöhr and Schwarting, 2009). We have previously reported that the same dose of morphine increased attention and effort in response to non-social reward cues (Eikemo et al., 2017), as well as enhancing visual exploration and gaze time to the eye region of faces with neutral expressions (Chelnokova et al., 2016). However, the current study did not measure motivation or collect behavioural measures that could perhaps have revealed similar changes in attention or social interest. We cannot conclude on the basis of the current findings that MOR agonism would not enhance approach behaviours or other evaluations of the stimuli, such as perceived friendliness (Laeng et al., 2010).

The present study asked participants to rate how angry (or happy) the person in the picture was, a question of magnitude that does not directly tap into affective impact of the stimuli. This type of rating is perhaps more akin to ratings of sensory intensity. MOR agonists are thought to target the affective rather than the sensory dimension of pain (Navratilova and Porreca, 2014). Indeed, the present pattern of results may stem from the use of rating scales requiring participants to report on the amount of an emotion they believed the face expressed, rather than e.g. reporting how friendly or approachable the depicted person seemed. Future studies assessing core impressions rather than perceived emotional expression, might find morphine-induced changes for explicitly happy and angry faces as well as for neutral or implicit expressions. 
At larger dosages, MOR drugs are also known for their ability to alter affective state (Comer and Zacny, 2010). While a state of anxiety is associated with heightened sensitivity to subtle threat cues (Mogg and Bradley, 2002), an increase in confidence induced by a non-sedative morphine dose was proposed to reduce threat sensitivity (Panksepp et al., 1985). In the current investigation, participants did not report feeling more confident or less anxious in the morphine condition. However, the dose of morphine was selected with the specific aim that it would produce changes in task behaviour without causing euphoria, subjective 'high' or other state changes. This design ensures that the effects of morphine are not compromised by potential expectation effects. Future studies should address whether the observed effects at a small, but analgesic dose of $\mu$-opioid agonist scale linearly with higher doses activating more receptors.

We have previously shown that $10 \mathrm{mg}$ morphine tablets can enhance motivated behaviour in humans without producing a corresponding change in self-reported subjective state (Chelnokova et al., 2016; Eikemo et al., 2017). We therefore speculate that the present effect of morphine on anger perception may reflect a subtle change in emotional-motivational state not immediately available for introspection, but with behavioural consequences consistent with increased confidence and a reduced motivation to explore potential threat cues in the environment. Future studies should include a broader range of measures to explicitly assess whether morphine's effects on anger perception are indeed related to such subtle changes in confidence or threat sensitivity. Further, the present design did not include direct or indirect measures of social motivation. Future studies should specifically address putative effects of opioid drugs on motivation to approach or avoid social stimuli such as the faces presented here.

Interestingly, participants' ability to detect a difference between faces showing implicitly happy and angry expressions was unaltered by morphine treatment. Anger perception was dampened to a 
comparable degree across implicitly angry, neutral and implicitly happy stimuli. Accordingly, the alternative hypothesis that morphine would improve detection of implicit facial expressions regardless of valence was not supported by the current results. Based on our previous finding that morphine increased visual exploration of faces as well as time spent looking at the eye-region of neutral faces, whereas opioid blockade had the opposite effect (Chelnokova et al., 2016), we reasoned that morphine could enhance sensitivity to cues of implicit emotional expressions. This would have been parallel to effects observed with intranasal oxytocin, which also increased gaze towards the eyes (Guastella et al., 2008). Oxytocin administration often improves emotion recognition (Leppanen et al., 2017), as we have previously reported for the implicit and explicit expressions used in the present design (Leknes et al., 2013).

Since oxytocin-mediated improvement of emotion recognition is reported particularly for individuals less proficient in detecting subtle emotional cues (Bartz and Hollander, 2008; Leknes et al., 2013), we also explored whether a similar enhancement in sensitivity to cues of both anger and happiness occurred with morphine in participants with higher autism trait scores. Autism spectrum disorder has been linked to opioid system disruptions in several rodent studies, and opioid dysregulation is specifically proposed to underpin the blunted social reward responses associated with ASD in humans (Pellissier et al., 2017). In mice, an intact MOR system is necessary for development of social reward processing, as evidenced by genetic knock-out mice lacking the MOR gene altogether (OPRM-

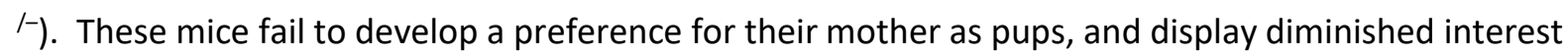
in social reward cues throughout development (Wöhr et al., 2011). In the current study, we found no significant effect of $A Q$ on emotion perception and no significant interaction between $A Q$ scores and drug condition. Furthermore, we found no evidence that $A Q$ scores covaried with emotion sensitivity (ES), a measure of participants' ability to correctly differentiate between implicitly angry and implicitly happy expressions. This null finding could be down to insufficient variance in $E S$ and $A Q$ measures. None of the participants in the current study were diagnosed with ASD; AQ scores were 
within the range found in other healthy control samples (Baron-Cohen et al., 2001b); and ES scores indicated a limited spread around the mean. Hence, we cannot conclude from this that opioid drugs could not improve emotion perception in clinical populations.

\section{Conclusion}

Compared to placebo, morphine reduced perception of anger in faces with implicitly angry, implicitly happy and neutral facial expressions, without affecting perception of anger in explicitly angry faces or overall perception of happiness. The effect of morphine on anger perception occurred at a low dose of the drug, and was statistically unrelated to subjective drug effects, mood, autism trait and gender. These results are in line with hypotheses that MOR activation increases social confidence and reduces sensitivity to threat cues, but contrast with previous reports of MOR stimulation leading to enhanced positive responses to social stimuli indicative of reward. Future studies testing this hypothesis should include a broader range of measures of confidence and threat sensitivity as well as direct assessment of motivation to approach or avoid social stimuli with different emotional content.

\section{Funding and Disclosure}

This work was supported by grant number 2014100 from the South-Eastern Norway Regional Health Authority to TH/SL. The authors have no conflicts of interest.

\section{Acknowledgements}

We would like to thank Selma Lie and Andreas Dahl for help with piloting and data collection. 


\section{Bibliography}

Baron-Cohen, S., Wheelwright, S., Hill, J., Raste, Y., Plumb, I., 2001a. The "Reading the Mind in the Eyes" Test revised version: A study with normal adults, and adults with Asperger syndrome or highfunctioning autism. Journal of Child Psychology and Psychiatry and Allied Disciplines 42, 241-251.

Baron-Cohen, S., Wheelwright, S., Skinner, R., Martin, J., Clubley, E., 2001b. The autism-spectrum quotient (AQ): evidence from Asperger syndrome/high-functioning autism, males and females, scientists and mathematicians. Journal of autism and developmental disorders 31, 5-17.

Bartz, J.A., Hollander, E., 2008. Oxytocin and experimental therapeutics in autism spectrum disorders. Prog Brain Res 170, 451-462.

Bartz, J.A., Zaki, J., Bolger, N., Hollander, E., Ludwig, N.N., Kolevzon, A., Ochsner, K.N., 2010. Oxytocin Selectively Improves Empathic Accuracy. Psychological Science 21, 1426-1428.

Berman, A.H., Bergman, H., Palmstierna, T., Schlyter, F., 2005. Evaluation of the Drug Use Disorders Identification Test (DUDIT) in criminal justice and detoxification settings and in a Swedish population sample. European addiction research 11, 22-31.

Berridge, K.C., Kringelbach, M.L., 2013. Neuroscience of affect: brain mechanisms of pleasure and displeasure. Current opinion in neurobiology 23, 294-303.

Bershad, A.K., Jaffe, J.H., Childs, E., de Wit, H., 2015. Opioid partial agonist buprenorphine dampens responses to psychosocial stress in humans. Psychoneuroendocrinology 52, 281-288.

Bershad, A.K., Seiden, J.A., de Wit, H., 2016. Effects of buprenorphine on responses to social stimuli in healthy adults. Psychoneuroendocrinology 63, 43-49.

Calvo, M.G., Lundqvist, D., 2008. Facial expressions of emotion (KDEF): identification under different display-duration conditions. Behav Res Methods 40, 109-115.

Chelnokova, O., Laeng, B., Eikemo, M., Riegels, J., Løseth, G., Maurud, H., Willoch, F., Leknes, S., 2014. Rewards of beauty: The opioid system mediates social motivation in humans. Molecular Psychiatry 19, 746-747.

Chelnokova, O., Laeng, B., Løseth, G., Eikemo, M., Willoch, F., Leknes, S., 2016. The $\mu$-opioid system promotes visual attention to faces and eyes. Social Cognitive and Affective Neuroscience.

Chevallier, C., Kohls, G., Troiani, V., Brodkin, E.S., Schultz, R.T., 2012. The Social Motivation Theory of Autism. Trends in Cognitive Sciences 16, 231-239.

Comer, S.M., Zacny, J.P., 2010. Subjective Effects of Opioids, Mind-Altering Drugs: The Science of Subjective Experience.

Dum, J., Herz, A., 1984. Endorphinergic modulation of neural reward systems indicated by behavioral changes. Pharmacol Biochem Behav 21, 259-266.

Eikemo, M., Biele, G., Willoch, F., Thomsen, L., Leknes, S., 2017. Opioid Modulation of Value-Based Decision-Making in Healthy Humans. Neuropsychopharmacology 42, 1833-1840. 
Eikemo, M., Løseth, G., Johnstone, T., Gjerstad, J., Willoch, F., Leknes, S., 2016. Sweet taste pleasantness is modulated by morphine and naltrexone. Psychopharmacology (Berlin).

Eippert, F., Bingel, U., Schoell, E., Yacubian, J., Buchel, C., 2008. Blockade of Endogenous Opioid Neurotransmission Enhances Acquisition of Conditioned Fear in Humans. J. Neurosci. 28, 5465-5472.

Fields, H.L., 2006. A Motivation-Decision Model of Pain: The Role of Opioids, in: Flor, H., Kalso, E., Dostrovsky, J.O. (Eds.), Proceedings of the 11th World Congress on Pain. IASP Press, Seattle, pp. 449459.

Giovannoni, G., Van Schalkwyk, J., Fritz, V.U., Lees, A.J., 1999. Bradykinesia akinesia incoordination test (BRAIN TEST): An objective computerised assessment of upper limb motor function. Journal of Neurology, Neurosurgery and Psychiatry 67, 624-629.

Guastella, A.J., Mitchell, P.B., Dadds, M.R., 2008. Oxytocin Increases Gaze to the Eye Region of Human Faces. Biological Psychiatry 63, 3-5.

Haaker, J., Yi, J., Petrovic, P., Olsson, A., 2017. Endogenous opioids regulate social threat learning in humans. Nature Communications 8.

Hsu, D.T., Sanford, B.J., Meyers, K.K., Love, T.M., Hazlett, K.E., Wang, H., Ni, L., Walker, S.J., Mickey, B.J., Korycinski, S.T., Koeppe, R.A., Crocker, J.K., Langenecker, S.A., Zubieta, J.K., 2013. Response of the [mu]-opioid system to social rejection and acceptance. Molecular Psychiatry 18, 1211-1217.

Ipser, J.C., Terburg, D., Syal, S., Phillips, N., Solms, M., Panksepp, J., Malcolm-Smith, S., Thomas, K., Stein, D.J., van Honk, J., 2013. Reduced fear-recognition sensitivity following acute buprenorphine administration in healthy volunteers. Psychoneuroendocrinology 38, 166-170.

Itier, R.J., Batty, M., 2009. Neural bases of eye and gaze processing: the core of social cognition. Neuroscience \& Biobehavioral Reviews 33, 843-863.

Laeng, B., Profeti, I., Sæther, L., Adolfsdottir, S., Lundervold, A.J., Vangberg, T., Øvervoll, M., Johnsen, S.H., Waterloo, K., 2010. Invisible expressions evoke core impressions. Emotion 10, 573-586.

Leknes, S., Tracey, I., 2008. A common neurobiology for pain and pleasure. Nat Rev Neurosci 9, 314320.

Leknes, S., Wessberg, J., Ellingsen, D.M., Chelnokova, O., Olausson, H., Laeng, B., 2013. Oxytocin enhances pupil dilation and sensitivity to 'hidden' emotional expressions. Social Cognitive and Affective Neuroscience 8, 741-749.

Leppanen, J., Ng, K.W., Tchanturia, K., Treasure, J., 2017. Meta-analysis of the effects of intranasal oxytocin on interpretation and expression of emotions. Neuroscience and Biobehavioral Reviews 78, 125-144.

Levine, J.D., Gordon, N.C., Fields, H.L., 1978. The mechanism of placebo analgesia. Lancet 2, 654-657.

Lugo, R.A., Kern, S.E., 2002. Clinical Pharmacokinetics of Morphine. Journal of Pain and Palliative Care Pharmacotherapy 16, 5-18. 
Lundqvist, D., Flykt, A., Ohman, A., 1998. Karolinska Directed Emotional Faces [Database of standardized facial images], in: Psychology Section, D.o.C.N., Karolinska Hospital, S-171 76 Stockholm, Sweden (Ed.).

Lutz, P.E., Kieffer, B.L., 2013. The multiple facets of opioid receptor function: implications for addiction. Current opinion in neurobiology.

Løseth, G., Ellingsen, D.M., Leknes, S., 2014. State-dependent $\mu$-opioid modulation of social motivation. Frontiers in Behavioral Neuroscience 8.

Meier, I.M., Bos, P.A., Hamilton, K., Stein, D.J., van Honk, J., Malcolm-Smith, S., 2016. Naltrexone increases negatively-valenced facial responses to happy faces in female participants. Psychoneuroendocrinology 74, 65-68.

Mogg, K., Bradley, B.P., 2002. Selective orienting of attention to masked threat faces in social anxiety. Behaviour Research and Therapy 40, 1403-1414.

Navratilova, E., Porreca, F., 2014. Reward and motivation in pain and pain relief. Nature Neuroscience $17,1304-1312$.

Panksepp, J., Jalowiec, J., DeEskinazi, F.G., Bishop, P., 1985. Opiates and play dominance in juvenile rats. Behavioral Neuroscience 99, 441-453.

Pellissier, L.P., Gandía, J., Laboute, T., Becker, J.A.J., Le Merrer, J., 2017. $\mu$ opioid receptor, social behaviour and autism spectrum disorder: Reward matters. British journal of pharmacology.

Poljac, E., Poljac, E., Wagemans, J., 2013. Reduced accuracy and sensitivity in the perception of emotional facial expressions in individuals with high autism spectrum traits. Autism 17, 668-680.

Saunders, J.B., Aasland, O.G., Babor, T.F., De La Fuente, J.R., Grant, M., 1993. Development of the Alcohol Use Disorders Identification Test (AUDIT): WHO Collaborative Project on Early Detection of Persons with Harmful Alcohol Consumption-II. Addiction 88, 791-804.

Spreckelmeyer, K.N., Krach, S., Kohls, G., Rademacher, L., Irmak, A., Konrad, K., Kircher, T., Gründer, G., 2009. Anticipation of monetary and social reward differently activates mesolimbic brain structures in men and women. Social Cognitive and Affective Neuroscience 4, 158-165.

Syal, S., Ipser, J., Terburg, D., Solms, M., Panksepp, J., Malcolm-Smith, S., Bos, P.A., Montoya, E.R., Stein, D.J., van Honk, J., 2015. Improved memory for reward cues following acute buprenorphine administration in humans. Psychoneuroendocrinology 53, 10-15.

Trezza, V., Damsteegt, R., Achterberg, E.J.M., Vanderschuren, L.J.M.J., 2011. Nucleus Accumbens $\mu-$ Opioid Receptors Mediate Social Reward. The Journal of Neuroscience 31, 6362-6370.

Vanderschuren, L.J.M.J., Niesink, R.J.M., Spruijt, B.M., Van Ree, J.M., 1995a. Effects of morphine on different aspects of social play in juvenile rats. Psychopharmacology 117, 225-231.

Vanderschuren, L.J.M.J., Niesink, R.J.M., Spruijt, B.M., Van Ree, J.M., 1995b. Influence of environmental factors on social play behavior of juvenile rats. Physiology and Behavior 58, 119-123.

Vanderschuren, L.J.M.J., Spruijt, B.M., Hol, T., Niesink, R.J., Van Ree, J.M., 1996. Sequential analysis of social play behavior in juvenile rats: Effects of morphine. Behavioural Brain Research 72, 89-95. 
Vindenes, V., Handal, M., Ripel, Å., Boix, F., Mørland, J., 2006. Conditioned place preference induced by morphine and morphine-6-glucuronide in mice. Pharmacology Biochemistry and Behavior 85, 292297.

Wardle, M.C., Bershad, A.K., de Wit, H., 2016. Naltrexone alters the processing of social and emotional stimuli in healthy adults. Social Neuroscience 11, 579-591.

Wöhr, M., Moles, A., Schwarting, R.K.W., D'Amato, F.R., 2011. Lack of social exploratory activation in male $\mu$-opioid receptor KO mice in response to playback of female ultrasonic vocalizations. Social Neuroscience 6, 76-87.

Wöhr, M., Schwarting, R.K.W., 2009. Ultrasonic communication in rats: Effects of morphine and naloxone on vocal and behavioral responses to playback of $50-\mathrm{kHz}$ vocalizations. Pharmacology Biochemistry and Behavior 94, 285-295. 